\title{
Carotid cavernous fistula
}

\section{SD Mathebula}

Department of Optometry, University of Limpopo, Private Bag X1106, Sovenga, 0727 South Africa

<solanim@ul.ac.za>

The cavernous sinuses are a paired structure within the sphenoid bone in the anterior portion of the middle cranial fossa. ${ }^{1-7}$ These sinuses extend anteriorly from the superior orbital fissure to the petrous portion of the temporal bone separately. The cavernous sinuses communicate with each other via the anterior and posterior intercavernous sinus, also known as the circular sinus. The anatomy of the cavernous sinus is truly unique because it is the only anatomic location in the body in which an artery travels completely through a venous structure. The cavernous sinus essentially functions as a dural venous structure, receiving blood supply from the superior and inferior ophthalmic veins. After entering the sinus, the venous blood is drained via the sphenoparietal sinus, the superior petrosal sinus, the basilar plexus and the pterygoid plexus. The venous architecture of the cavernous sinus is maintained by multiple communicating sinusoids, allowing the venous blood flow to be quite slow and of low pressure.

After bifurcating from the common carotid artery in the anterior cervical triangle, the internal carotid artery enters the skull via the foramen lacerum and the carotid canal. ${ }^{1-7}$ It continues through the petrosal canal and enters the cavernous sinus on the medial aspect of the sinus. Within the cavernous sinus, the internal carotid artery is bound by strong dural filaments and attachments, especially at its entrance and exit by its inferior and superior ascending segments. The siphon portion of the internal carotid artery is the sshaped or sigmoid segment that runs through the cavernous sinus. The meningohypophyseal trunk and the inferior cavernous sinus arteries are the largest branches of the intracavernous portion of the internal carotid artery. Smaller arterial branches also arise from the internal carotid artery within the sinus, forming intracavernous carotid plexus. The intracavernous carotid artery can be considered to be composed of three continuous segments: the intracavernous carotid artery enters the sinus as the posterior ascending segment, then the artery turns anteriorly, where it becomes the horizontal segment and finally, the artery turns superiorly, where it becomes the anterior ascending segment. The internal carotid artery leaves the cavernous sinus underneath the anterior clinoid process of the lesser wing of the sphenoid bone. Because the internal carotid artery is fixed to the surrounding dura of the base of the skull, it is exposed to shearing forces and penetrating injuries. Other clinically significant structures within the cavernous sinus are cranial nerves III (oculomotor), IV (trochlear), V1 (ophthalmic division of trigeminal), V2 (maxillary division of trigeminal) and VI (abducens). This anatomical configuration is clinically relevant when evaluating patients with carotid cavernous fistula.

Carotid cavernous fistula (CCF) is an abnormal communication between the carotid arterial system and the venous cavernous sinus. ${ }^{4-10}$ In most instances, the abnormality is between the internal carotid artery and the cavernous fistula. Benjamin Taverns first described this condition in 1809 as unilateral pulsating exophthalmos in a patient who subsequently lost vision in the affected eye. ${ }^{5}$ Since that time much has been learned of the cause, classification, anatomy and treatment.

The abnormal communication results in high-pressure arterial blood entering the low-pressure venous cavernous sinus, which interferes with the normal patterns of venous drainage and which compromises blood flow into the cavernous sinus and globe. ${ }^{7}, 10$ The restriction of normal egress of blood from the cavernous sinus may result in redirection of venous flow into the cortical veins or other venous structures with the development of complications, such as venous infarct.

Fundamentally, CCFs are classified as direct or indirect, traumatic or spontaneous and high or low flow. ${ }^{5}$ The classification scheme established by Barrow et $a l^{2}$ is used frequently. It divides CCFs into four angiographic types. Type A fistulas are direct communications between the internal carotid artery and the 
cavernous sinus. Types B, C and D are indirect shunts (dural) because fistulas to the cavernous sinus arise from dural arteries and not directly from the internal carotid artery. Type A is more common in young males. ${ }^{9}$ Types B, C and D are more common in women older than 50 years, with a 7:1 female-to-male ratio. ${ }^{9}$

Direct CCFs (type A) result from a direct connection between the cavernous segment of the intracavernous carotid artery and the cavernous sinus itself. ${ }^{7-9}$ Indirect CCFs arise from abnormal shunts to the cavernous sinus from the meningeal branches of the intracavernous carotid artery (type B), from the meningeal branches of the external carotid artery (type $\mathrm{C}$ ) and from the meningeal branches of both the intracavernous carotid artery and the external carotid artery (type D). Types B, C and D tend to be low-flow and lower pressure fistulas with a slower progression of signs and symptoms. ${ }^{7-9}$

Traumatic CCFs may occur after blunt head injuries in which the intracavernous carotid artery is torn. ${ }^{7-13}$ Penetrating head injury can lead to fistula formation by direct laceration of intracavernous vessels. Direct CCFs usually occur suddenly, resulting from a tear in the arterial wall connecting the internal carotid artery to the cavernous sinus. ${ }^{7-10}$ The fistulas typically have a high flow rate. Indirect CCFs have a more gradual onset over days, weeks, or months, a less fulminating course and generally lower flow. ${ }^{2,11}$

Spontaneous CCFs usually result from a ruptured carotid aneurysm. However, some authors have hypothesized that these fistula formation has been associated with fibromuscular dysplasia, collagen vascular disease, Ehlers-Danlos syndrome, antherosclerotic vascular disease, hypertension or straining. ${ }^{2,11}$

Irrespective of the etiology of CCF, shunting of the blood between a high-flow arterial system and a lowflow venous system produces increased vascular pressure and resistance in the venous system. This increased venous pressure impedes the venous drainage of the cavernous sinus, thereby causing vascular congestion in the regions that are normally drained by the cavernous sinus. This congestion within and around the cavernous sinus accounts for the clinical symptoms.

The orbits, whose venous drainage travels to the cavernous sinuses through the superior and inferior ophthalmic veins, are the first structures to manifest the symptoms of this reversed blood flow. ${ }^{7-9}$ Patients often complain of a swollen red eye, orbital pain, diplopia, headache and progressive vision loss. ${ }^{7}$ Loss of vision is the most common serious problem associated with posttraumatic CCF. Vision loss can result from corneal exposure and changes in retinal circulation, but the major treat to vision is from hypoxia. ${ }^{10}$ Decreased mean arterial pressure with increased venous pressure reduces the ocular perfusion pressure. The subsequent ischemic damage may be irreversible.

Common clinical signs include proptosis, orbital bruit, chemosis, extraocular palsy, pulsating exophthalmos, ptosis, elevated intraocular pressure, anterior segment ischaemia and retinopathy.

The clinical manifestations of traumatic CCF depend on the venous drainage of the fistula. In most cases, there is mixed anterior and posterior drainage by means of the ophthalmic venous system and the inferior petrosal sinus. This results in the syndrome of bruit, chemosis, proptosis and visual impairment with or without cranial nerve involvement. ${ }^{9-13}$ Bruit is the most consistent finding in association with hyperemic conjunctivitis. Arterialization of sclera and orbital veins causes chemosis and proptosis. ${ }^{13}$ Oculomotor palsies and diplopia commonly occur secondary to mechanical restriction from increased orbital mass and from direct compression of cranial nerves III, IV and VI. The abducens nerve is the most vulnerable because it runs within the sinus itself. ${ }^{9}$

Unlike direct fistulas, indirect fistulas have a gradual onset; with generally milder presentation. ${ }^{2}$ They often do not demonstrate the classic triad of symptoms. Patients with these fistulas usually have chronically red eyes as a result of tortuous arterialization of the conjunctiva. Often, no ocular bruit is associated with these fistulas.

Diagnosis of traumatic CCF is usually straight forward, although the majority of indirect CCFs are not diagnosed for weeks or even months after the onset of symptoms. ${ }^{2,-11}$ Because of the traumatic cause, patients often have associated injuries at multiple levels and a thorough clinical assessment is warranted. Angiography ${ }^{10}$ is the current gold standard in the diagnosis of CCF. Computerized tomography (CT) scan of the head is useful because it can depict any bony fracture. It can even reveal the precise location and size of CCF. In addition to angiography and CT, magnetic resonance imaging is recommended. Complete ophthalmic workup is indicated and should include gonioscopy, ophthalmoscopy, as well as an assessment of visual acuity, pupillary function and intraocular pressure. 
Symptomatic direct CCFs (type A) spontaneously resolve only in rare cases. Therefore, they almost always require urgent treatment. The goal of treatment is to eliminate flow through the fistula but also to maintain internal carotid patency. Indirect (dural) CCFs (types B, C and D) sometimes resolve spontaneously. Treatment is indicated in patients with dural CCFs who present with proptosis, visual loss, palsy of cranial nerve VI, neovascularization of the iris or retina, severe elevated IOP, intractable bruit or severe pain. As many as $90 \%$ of patients with direct CCFs may lose vision in the absence of treatment. ${ }^{8}$ Of patients with untreated indirect CCFs, $20-30 \%$ experience loss of vision. ${ }^{8}$

Goals of treatment include preservation of vision with resolution of associated symptoms while avoiding cerebral ischaemic complications. The ultimate and definitive treatment of CCFs falls beyond the scope of optometry. Nevertheless, clinicians treating patients with craniofacial injuries should have a complete understanding of this pathological entity. Treatment is performed by interventional radiologists and neurologic surgeons.

\section{References}

1. Phillips PH. Carotid-cavernous fistulas. Neurosurg Clin N Am 199910 653-665.

2. Barrow DL, Spector RH and Braun IF. Classification and treatment of spontaneous carotid-cavernous sinus fistulas. J Neurosurg $1985 \mathbf{6 2}$ 248-256.

3. Yanoff M. Ophthalmology. St Louis, MO: Mosby; 2004 1403-1404.

4. Jimenez DF and Gibbs SR. Carotid-cavernous sinus fistula in craniofacial trauma: classification and treatment. J Craniomaxillo Trauma 19973 9-18.

5. Niamtu J and Campbell RL. Carotid cavernous fistula. J Oral Maxillofac Surg 198240 52-56.

6. Purvin V. Cerebrovascular disease and the visual system. Ophthalmol Clin North Am 200417 329-355.

7. Low HL, Sawlani V and Redfern RM. Carotid-cavernous fistula after blunt traumatic to the neck. Br J Neurosurg $200620254-259$.

8. Wen L, Yang X, Liu W, Pan D, Shen G, Zheng X, Xu X and Jiang D. Bilateral traumatic carotid cavernous fistula: the manifestation, transvascular embolization and prevention of the vascular complications after therapeutic embolization. J Craniofac Surg 2007 18 74-77.

9. Fabian TS, Woody JD and Ciraulo DL. Posttraumatic carotid cavernous fistula: frequency analysis of signs, symptoms, and disability outcomes after angiographic embolization. J Trauma 199947 275-281.

10. Hu WY and Hudon M. Neuroimaging highlight: traumatic carotid-cavernous fistula. Can J Neurol Sci 2000 27 71-72.

11. Uehara T, Tabuchi M, Kawaguchi T and Mori E. Spontaneous dural carotid cavernous sinus fistula presenting isolated ophthalmoplegia: evaluation with MR angiography. Neurology $1998 \mathbf{5 0} 814-816$.

12. Zachariades N and Papavassiliou D. Traumatic carotid-cavernous sinus fistula. J Craniomaxillofac Surg 1988 16 385-388.

13. Fattahi TT, Brandt MT, Jenkins WS and Steinberg B. Traumatic carotid-cavernous fistula: pathophysiology and treatment. $J$ Craniofac Surg $200314240-246$. 